\title{
UNIVALENCE OF BESSEL FUNCTIONS
}

R. K. BROWN

1. Introduction. In 1954 M. S. Robertson [2] obtained sufficient conditions for the univalence in the unit circle of functions

$$
[W(z)]^{1 / \alpha}=\left[z^{\alpha} \sum_{n=0}^{\infty} a_{n} z^{n}\right]^{1 / \alpha}, \quad a_{0}=1,
$$

where $R\{\alpha\} \geqq 1 / 2$ and $W(z)$ is a solution of the differential equation

$$
W^{\prime \prime}(z)+p(z) W(z)=0, \quad|z|<1 .
$$

In this paper we employ the methods of Robertson to obtain information concerning the univalence of the functions $[T(z)]^{1 / \nu}(\nu \neq 0)$ and $z^{1-\nu} T(z)$ where

$$
T(z)=z^{\nu} \sum_{n=0}^{\infty} a_{n} z^{n}, \quad R\{\nu\} \geqq 0,
$$

is a solution of the differential equation

$$
T^{\prime \prime}(z)+\frac{1}{z} T^{\prime}(z)+q(z) T(z)=0, \quad|z|<R .
$$

In particular we shall first determine a radius of univalence for the normalized Bessel functions $\left[J_{\nu}(z)\right]^{1 / \nu}$ for values of $\nu$ belonging to the region $G$ defined by the inequalities $R\{\nu\}>0,|\arg \nu|<\pi / 4$. Then we shall determine the radius of univalence of the functions $z^{1-\nu} J_{\nu}(z)$ for values of $\nu$ belonging to a subset of the closure of $G$. When $\nu$ is real and positive we shall determine the exact radius of star-likeness of both of the above-mentioned classes of normalized Bessel functions.

Our results concerning the functions $z^{1-\nu} J_{\nu}(z)$ "sharpen" those of Kreyszig and Todd [1] when $\nu \geqq 0$ and extend their results for complex values of $\nu$.

2. Preliminaries. Let

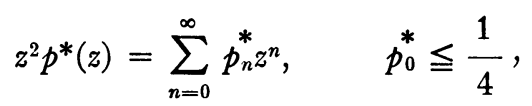

be regular for $|z|<R$ and real on the real axis. Given any nonnegative constant $C$, define

Presented to the Society, September 3, 1959; received by the editors March 10, 1959 and, in revised form, July 8, 1959. 


$$
W_{C}(z)=z^{\alpha^{*}} \sum_{n=0}^{\infty} a_{n}^{*}(C) z^{n}, \quad a_{0}^{*}(C)=1,
$$

to be the unique solution for $|z|<R$ of the differential equation

$$
W^{\prime \prime}(z)+\left\{C\left[p^{*}(z)-p_{0}^{*} / z^{2}\right]+p_{0}^{*} / z^{2}\right\} W(z)=0
$$

where $\alpha^{*}$ is the larger root of the associated indicial equation.

We now are ready to state the following

Lemma. Let $y(\rho), d y(\rho) / d \rho=y^{\prime}(\rho)$ be real functions, continuous in the real variable $\rho$ for $0<\rho<R$. For small values of $\rho$ let

Then

$$
y(\rho)=O\left(\rho^{\delta}\right), \quad y^{\prime}(\rho)=O\left(\rho^{\delta-1}\right), \quad \delta>1 / 2 .
$$

$$
\begin{aligned}
\int_{0}^{r}\left\{C \left[\rho^{2} p^{*}(\rho)\right.\right. & \left.\left.-p_{0}^{*}\right]+p_{0}^{*}\right\} y^{2}(\rho) \frac{d \rho}{\rho^{2}} \\
& \leqq \int_{0}^{r}\left[y^{\prime}(\rho)\right]^{2} d \rho-\frac{W_{C}^{\prime}(r)}{W_{C}(r)} \cdot y^{2}(r), \quad 0<r<R,
\end{aligned}
$$

where $W_{C}(z)$ is the solution (2.2) of (2.3).

The proof of this lemma is with obvious modifications the same as that given by Robertson [2] for the case $R=1$. We will not reproduce it here.

With the aid of the lemma we are able to prove the following THeOREM 1. Let $z^{2} p(z)$ be regular for $|z|<R$ and satisfy the inequality

$$
\mathcal{R}\left\{e^{i \gamma} z^{2} p(z)\right\} \leqq \cos \gamma\left\{C\left[|z|^{2} p^{*}(|z|)-p_{0}^{*}\right]+p_{0}^{*}\right\}
$$

where $C \geqq 0,|\gamma| \leqq \pi / 2$, and $z^{2} p^{*}(z)$ is defined in (2.1). With $p(z)$ chosen in this manner we define

$$
W(z)=z^{\alpha} \sum_{n=0}^{\infty} a_{n} z^{n}, \quad a_{0}=1,|z|<R,
$$

to be the unique solution of (1.1) corresponding to the root with larger real part of the associated indicial equation. Let $W_{C}(z)$ be defined as in (2.2). Then

$$
R\left\{e^{i \gamma} \frac{z W^{\prime}(z)}{W(z)}\right\} \geqq|z| \frac{W_{C}^{\prime}(|z|)}{W_{C}(|z|)} \cos \gamma
$$

for all $|z| \leqq \rho<R$.

Proof. If in (2.6) we have $R\{\alpha\}>1 / 2$ then 


$$
\begin{aligned}
|W(z)|^{2} \Re & \left\{e^{i \gamma} \frac{z W^{\prime}(z)}{W(z)}\right\} \\
& =r \cos \gamma \int_{0}^{r}\left|W^{\prime}\right|^{2} d \rho-r \cdot \int_{0}^{r} \Re\left\{e^{i \gamma} z^{2} p(z)\right\}_{|z|=\rho} \frac{|W|^{2}}{\rho^{2}} d \rho
\end{aligned}
$$

for all $0 \leqq r<R$.

This equation is known as the "Green's transform" of (1.1) and in the form (2.8) is due to Robertson [2]. The inequality (2.7) now follows immediately from (2.8), (2.5), and (2.4) with $y(\rho)=W(\rho)$.

The proof for the case when $R\{\alpha\}=1 / 2$ in (2.6) follows from the continuity of $z W^{\prime}(z) / W(z)$ as a function of $\alpha$ for $R\{\alpha\}>0$ (see [2, p. 258]).

We conclude this section with the definitions of the terms "starlike" and "spiral-like."

Definition. A function $f(z)=\sum_{n=1}^{\infty} a_{n} z^{n}, a_{1} \neq 0$, regular for $|z|<R$ will be called spiral-like in $|z|<R$ if for some real constant $\gamma(|\gamma| \leqq \pi / 2)$ the function $f(z)$ satisfies the inequality

$$
\Re\left\{e^{i \gamma} \frac{z f^{\prime}(z)}{f(z)}\right\} \geqq 0
$$

for all $|z|<R$. In the special case when $\gamma=0$ we say that $f(z)$ is star-like with respect to the origin in $|z|<R$.

It was shown by Špaček [3] that (2.9) is sufficient for the univalence in $|z|<R$ of $f(z)$ whenever $f^{\prime}(0) \neq 0$.

3. Bessel's equation. In this section we state our two theorems concerning the univalence of normalized solutions of Bessel's equation

$$
T^{\prime \prime}(z)+\frac{1}{z} T^{\prime}(z)+\left(1-\frac{\nu^{2}}{z^{2}}\right) T(z)=0, \quad|z|<R .
$$

THEOREM 2. Let the complex number $\nu$ satisfy the inequalities $R\{\nu\}$ $>0,|\arg \nu|<\pi / 4$. Then the normalized Bessel function $\left[J_{\nu}(z)\right]^{1 / \nu}$ is regular, univalent, and spiral-like in every circle $|z|=r<\rho_{\mu}$ where $\mu^{2}=R\left\{\nu^{2}\right\}, \mu>0$, and $\rho_{\mu}$ is the smallest positive zero of the function $J_{\mu}^{\prime}(r)$. In the particular case when $\nu$ is real and positive the function $\left[J_{\nu}(z)\right]^{1 / \nu}$ is star-like in $|z|<\rho_{\mu}$ but is not univalent in any larger circle.

TheOREM 3. Let the complex number $\nu=x+i y$ satisfy one of the following conditions:

$$
\begin{aligned}
& 0 \leqq x<1 \quad \text { and } y \leqq x \\
& x \geqq 1 \text { and } y^{2}<2 x-1 .
\end{aligned}
$$


Then the normalized Bessel function $z^{1-\nu} J_{\nu}(z)$ is regular, univalent, and spiral-like in every circle $|z|=r<\rho_{\mu}^{*}$ where $\mu^{2}=R\left\{\nu^{2}\right\}, \mu>0$, and $\rho_{\mu}^{*}$ is the smallest positive zero of the function $r J_{\mu}^{\prime}(r)+R\{1-\nu\} J_{\mu}(r)$. In the particular case when $\nu$ is real the function $z^{1-\nu} J_{\nu}(z)$ is univalently star-like in $|z|<\rho_{\mu}^{*}$ but is not univalent in any larger circle.

4. Proof of Theorem 2. Select any $\nu$ satisfying the inequalities $\Re\{\nu\}>0,|\arg \nu|<\pi / 4$, and consider (2.3) with $\mu^{2}=R\left\{\nu^{2}\right\}, C=1$, and

$$
z^{2} p^{*}(z)=z^{2}+1 / 4-\mu^{2} .
$$

In this manner we obtain the differential equation

$$
W^{\prime \prime}(z)+\left[1-\frac{1}{z^{2}}\left(\mu^{2}-1 / 4\right)\right] W(z)=0
$$

whose solution $W_{1}(z)$ as defined in (2.2) is

$$
W_{1}(z)=2^{\mu} \Gamma(\mu+1) z^{1 / 2} J_{\mu}(z) .
$$

Next, by setting

$$
z^{2} p(z)=z^{2}+1 / 4-\mu^{2}
$$

in (1.1) we find that the solution $W(z)$ of $(1.1)$ as defined in (2.1) is

$$
W(z)=2^{\nu} \Gamma(\nu+1) z^{1 / 2} J_{\nu}(z) .
$$

The solutions given in (4.3) and (4.5) are valid for all finite $z$. Moreover, $z^{2} p^{*}(z)$ and $z^{2} p(z)$ as given in (4.1) and (4.4) satisfy (2.7) for all finite values of $z$ when $\gamma=0$ and $C=1$. Therefore, from (2.7) it follows that

$$
\Re\left\{\frac{z W^{\prime}(z)}{W(z)}\right\} \geqq|z| \frac{W_{1}^{\prime}(|z|)}{W_{1}(|z|)}
$$

for all finite values of $z$. Thus, from (4.3), (4.5), and (4.6) we have

$$
\Re\left\{\frac{z J_{\nu}^{\prime}(z)}{J_{\nu}(z)}\right\} \geqq \frac{r J_{\mu}^{\prime}(r)}{J_{\mu}(r)}, \quad|z|=r
$$

for all finite $r$.

Since $\mu$ is positive it follows from (4.7) that

$$
R\left\{\frac{z J_{\mu}^{\prime}(z)}{J_{\mu}(z)}\right\} \geqq 0, \quad|z| \leqq \rho_{\mu},
$$

where $\rho_{\mu}$ is the smallest positive zero of $J_{\mu}^{\prime}(r)$. 
We now define

$$
F_{\nu}(z)=\left[J_{\nu}(z)\right]^{1 / \nu}
$$

where $\left[J_{\nu}(z)\right]^{1 / \nu}=(1 / \nu) \exp \left\{\log J_{\nu}(z)\right\}$ and $\log$ represents the principal branch of the logarithm. Then,

$$
\Re\left\{\frac{z F_{\nu}^{\prime}(z)}{F_{\nu}(z)}\right\}=\Re\left\{\frac{z J_{\nu}^{\prime}(z)}{J_{\nu}(z)}\right\}
$$

and it follows from (4.8) that in every circle $|z|=r<\rho_{\mu}$ the function $F_{\nu}(z)$ is spiral-like if $\nu$ is complex and is star-like if $\nu$ is real and positive.

Clearly, since $J_{\mu}^{\prime}(z)$ vanishes for $z=\rho_{\mu}$ the function $\left[J_{\mu}(z)\right]^{1 / \mu}, \mu>0$, cannot be univalent in any circle $|z|=r>\rho_{\mu}$.

This completes the proof of Theorem 2 .

5. Proof of Theorem 3. If in the proof of Theorem 2 we replace $F_{\nu}(z)$ in $(4.9)$ by the function

$$
S_{\nu}(z)=z^{1-\nu} J_{\nu}(z), \quad R\{\nu\} \geqq 0,
$$

then since

$$
R\left\{\frac{z S_{\nu}^{\prime}(z)}{S_{\nu}(z)}\right\}=R\{1-\nu\}+R\left\{\frac{z J_{\nu}^{\prime}(z)}{J_{\nu}(z)}\right\}
$$

it follows from (4.7) that

$$
\mathcal{R}\left\{\frac{z S_{\nu}^{\prime}(z)}{S_{\nu}(z)}\right\} \geqq R\{1-\nu\}+\frac{z J_{\mu}^{\prime}(z)}{J_{\mu}(z)}
$$

for all finite $z(|z|=r)$. Then, since (3.2) and (3.3) imply that the right-hand member of (5.3) is positive for sufficiently small values of $r$, it follows that

$$
\Re\left\{\frac{z S_{\nu}^{\prime}(z)}{S_{\nu}(z)}\right\} \geqq 0, \quad|z| \leqq \rho_{\mu}^{*},
$$

where $\rho_{\mu}^{*}$ is the smallest positive zero of the function

$$
r J_{\mu}^{\prime}(r)+R\{1-\nu\} J_{\mu}(r) .
$$

For non-negative real values of $\nu$ the vanishing of $S_{\nu}^{\prime}(z)$ for $z=\rho_{\mu}^{*}$ precludes the possibility that $S_{\nu}(z)$ is univalent in any circle $|z|$ $=r>\rho_{\mu}^{*}$.

We note here that for non-negative real values of $\nu$ the $\rho_{\mu}^{*}$ of our Theorem 3 is precisely the $\rho_{\nu}$ of [1]. 
6. Remarks. We note that if $T(z)=z^{\nu} \sum_{n=0}^{\infty} a_{n} z^{n}, R\{\nu\} \geqq 0$, satisfies (1.2) for $|z|<R$, then the function

$$
W(z)=z^{1 / 2} T(z)=z^{\alpha} \sum_{n=0}^{\infty} a_{n} z^{n}, \quad \propto\{\alpha\} \geqq 1 / 2,
$$

satisfies (1.1) with $z^{2} p(z)=z^{2} q(z)+1 / 4$. Thus Theorem 1 is applicable to an entire class of functions satisfying (1.2). In particular, therefore, one could obtain results analogous to those of Theorems 2 and 3 for the modified Bessel functions $I_{\nu}(z)$.

Many other results could be obtained by judicious selection of the function $q(z)$ subject to the conditions of Theorem 1 .

\section{BIBLIOGRAPHY}

1. E. O. A. Kreyszig and J. Todd, The radius of univalence of Bessel functions I, Notices Amer. Math. Soc. vol. 5 (1958) p. 664.

2. M. S. Robertson, Schlicht solutions of $W^{\prime \prime}+p W=0$, Trans. Amer. Math. Soc. vol. 76 (1954) pp. 254-274.

3. Lad. Spaček, Contribution à la théorie des fonctions univalentes, Časopis Pěst. Mat. Fys. vol. 62 (1936) pp. 12-19.

U. S. Army Signal R/D Laboratory, Fort Monmouth, New Jersey 